\title{
Static Output Feedback Control Design for Descriptor Systems
}

\author{
Mohamed Yagoubi ${ }^{1,2}$ \\ ${ }^{1}$ (Institut de Recherche en Communications et Cybernétique de Nantes) IRCCyN, UMR CNRS, 6597 at 1 rue de la Noë, \\ B.P. 93101, 44432 Nantes Cedex 3, France \\ 2 (Ecole des Mines de Nantes) EMN, 4 rue Alfred Kastler La Chantrerie, 44307 Nantes, France \\ Correspondence should be addressed to Mohamed Yagoubi, myagoubi@emn.fr
}

Received 9 December 2009; Accepted 19 May 2010

Academic Editor: L. Z. Yu

Copyright () 2010 Mohamed Yagoubi. This is an open access article distributed under the Creative Commons Attribution License, which permits unrestricted use, distribution, and reproduction in any medium, provided the original work is properly cited.

The static output feedback (SOF) synthesis problem for descriptor systems is considered in this paper. LMI-based algorithms are proposed to find potentially structured SOF gains ensuring admissibility and even $\mathrm{H}_{\infty}$ performance of the closed-loop system. These algorithms are then used to propose a (descriptor) observer-based $\mathrm{H}_{\infty}$ controller design method. An alternative technique for determining such separated estimation/control structure, after the design step, is also proposed. Several numerical examples, throughout the paper, demonstrate the effectiveness of the proposed algorithms.

\section{Introduction}

The descriptor framework encompasses a wide class of systems [1]. The so-called descriptor representation describes the dynamic part, static part, and improper part of the system. Even in the nonsingular case, descriptor systems are very useful to manipulate physical models without losing a physical parameterization or to describe controller/filter implementation. Strict LMI characterizations for admissibility, $\mathrm{H}_{\infty}$ and $\mathrm{H}_{2}$ norms of descriptor systems are established in $[2-7]$.

The static output feedback (SOF) control problem plays a central role in control theory and applications (see, e.g., $[8,9]$ and references therein). Because of its generality, several LMI-based SOF design methods have been proposed for state-space systems over last decade whereas there are few papers discussing the SOF control problem for descriptor systems [10]. Some existing results on the subject deal with only the problem of the closed-loop system regularity or impulse immunity $[11,12]$.

In this paper, a novel coordinate-descent iterative numerical procedure that can synthesize a SOF, ensuring the admissibility of the closed-loop system, is derived in the continuous time setting. The proposed algorithm does not require any special decomposition of the considered descriptor system. Contrary to some existing iterative LMI algorithms (see, e.g., [13] or [14]) no additional variables are needed for the proposed method. The idea underlying this method can be seen as an extension to the descriptor case of some LMI-based algorithms previously proposed in [15] or [16] in the state-space case.

Furthermore, it is well known in control theory that an important problem in control arises when a specific structure on the overall control scheme is considered, especially when dealing with complex or distributed systems. Thus, we extend the proposed algorithms to deal with SOF $\mathrm{H}_{\infty}$ controllers with a fixed structure for descriptor systems. The algorithm developed here is used to compute local optimal solutions. Decomposition in consecutive subproblems of the initial problem, following the same lines as in [14] or [15], is adopted.

In addition, the observer-based $\mathrm{H}_{\infty}$ control problem for descriptor systems is considered in this paper as a direct application of the proposed approach.

This paper is organized as follows. some preliminary results on descriptor systems are recalled in Section 2. In Section 3, some LMI-based algorithms for ensuring the admissibility of closed-loop descriptor systems, via potentially structured SOF gains are presented. Then, in Section 4, the extension of these algorithms to the $\mathrm{H}_{\infty}$ control problem is given. Section 5 tackles the observer-based $\mathrm{H}_{\infty}$ control problem for descriptor systems. Some concluding remarks are given in Section 6. 
Notation. The notation $A>0$ (resp., $A \geq 0$ ) stands for $A$ definite positive (resp., semidefinite positive). The notation $\mathrm{He}\{A\}$ stands for $A^{T}+A$ and $\bullet$ for terms that are induced by symmetry. $I$ (resp., $\Gamma$ ) indicates the identity matrix (resp., the matrix with all entries set to 1 ) with proper dimensions. The symbol $\otimes$ denotes the direct product of matrices.

\section{Preliminaries}

Let us consider the descriptor system given by

$$
\begin{gathered}
E \dot{x}=A x+B_{1} w+B_{2} u, \\
z=C_{1} x, \\
y=C_{2} x,
\end{gathered}
$$

where $x \in \mathbb{R}^{n}$ is the descriptor variable, $w \in \mathbb{R}^{q}$ is the disturbance, $u \in \mathbb{R}^{m}$ is the control input, $z \in \mathbb{R}^{l}$ is the controlled output, and $y \in \mathbb{R}^{p}$ is the measured output. The matrix $E$ may be singular and we denote its rank by $\operatorname{rank}(E)=r \leq n$. It is known that systems having direct transmission paths from $w$ and $u$ to $z$ and $y$ can be transformed by augmenting the descriptor variable as pointed out for example in [5].

If $\operatorname{det}(s E-A) \neq 0$ for some complex numbers, then system (1) is said to be regular [1]. A regular system of the form (1) is said to be impulse-free if

$$
\operatorname{deg}(\operatorname{det}(s E-A))=\operatorname{rank}(E)
$$

Definition 1. System (1) is admissible if it is regular and has neither impulsive modes nor unstable finite modes.

As defined above, the admissibility of descriptor systems can be formulated as an LMI feasibility problem as stated in [17]. Let us introduce matrices $V, U \in \mathbb{R}^{n \times(n-r)}$ which are of full-column rank such that $E V=0$ and $E^{T} U=0$ and matrices $E_{L}, E_{R} \in \mathbb{R}^{n \times r}$ of full-column rank such that $E=$ $E_{L} E_{R}^{T}$.

Lemma 2 (see [18]). System (1) is admissible if and only if there exist a positive definite matrix $P \in \mathbb{R}^{n \times n}$ and a matrix $S \in \mathbb{R}^{(n-r) \times(n-r)}$ satisfying the LMI:

$$
H e\left\{A\left(P E^{T}+V S U^{T}\right)\right\}<0
$$

Lemma 3 (see [18]). For a given positive number $\gamma$, the system (1) is stable and

$$
\left\|C_{1}(s E-A)^{-1} B_{1}\right\|_{\infty}<\gamma
$$

holds if and only if there exist a positive definite matrix $P \in$ $\mathbb{R}^{n \times n}$ and a matrix $S \in \mathbb{R}^{(n-r) \times(n-r)}$ satisfying the LMI:

$$
\left[\begin{array}{cc}
H e\left\{A\left(P E^{T}+V S U^{T}\right)\right\}+B_{1} B_{1}^{T} & \left(P E^{T}+V S U^{T}\right)^{T} C_{1}^{T} \\
\bullet & -\gamma^{2} I
\end{array}\right]<0 .
$$

Lemma 4 (see [18]). Let $P \in \mathbb{R}^{n \times n}$ be symmetric such that $E_{R}^{T} P E_{R}>0$ and $S$ be nonsingular. Then, $P E^{T}+V S U^{T}$ is nonsingular and its inverse is expressed as

$$
\left(P E^{T}+V S U^{T}\right)^{-1}=Q E+U R V^{T},
$$

where $Q \in \mathbb{R}^{n \times n}$ is symmetric and $R \in \mathbb{R}^{(n-r) \times(n-r)}$ is nonsingular such that

$$
\begin{gathered}
\left(E_{R}^{T} P E_{R}\right)^{-1}=E_{L}^{T} Q E_{L}, \\
R=\left(U^{T} U\right)^{-1} S^{-1}\left(V^{T} V\right)^{-1} .
\end{gathered}
$$

\section{SOF Admissibility Control}

3.1. LMI-Based Algorithm for the SOF Admissibility Control Problem. In this section, the static output feedback (SOF) admissibility control for descriptor systems given by (1) is investigated. The following assumption is made on system (1).

Assumption 5. (i) ( $\left.E, A, B_{2}\right)$ is finite dynamics stabilizable and impulse controllable [1]. (ii) $\left(E, A, C_{2}\right)$ is finite dynamics detectable and impulse observable [1].

The SOF admissibility control problem is to find an SOF controller:

$$
u=F y
$$

where $F \in \mathbb{R}^{m \times p}$ such that the closed-loop system with $w=0$ given by

$$
E \dot{x}=\left(A+B_{2} F C_{2}\right) x
$$

is admissible.

According to Lemma 2, (10) is admissible if and only if there exist a positive definite matrix $P$ and a matrix $S$ such that the following condition:

$$
H e\left\{\left(A+B_{2} F C_{2}\right)\left(P E^{T}+V S U^{T}\right)\right\}<0
$$

holds.

Recall that the matrix $P$ is related with the finite dynamics behavior and the matrix $S$ is related with regularity and the impulsive behavior. The main point of an iterative LMI algorithm concerning the problem stated above is to find initial variables $P$ and $S$. In this note, Algorithm 6 will consider the problem of finding initializing variables, namely, $P_{s}$ and $S_{s}$. Then, Algorithm 7 will consider the problem of deriving a feasible solution $F$ of the SOF admissibility control problem stated above starting with the variables $P_{s}$ and $S_{s}$ derived by Algorithm 6 .

By setting: $K_{1}:=F C_{2} P$ and $K_{2}:=F C_{2} V S,(11)$ becomes

$$
H e\left\{A\left(P E^{T}+V S U^{T}\right)+B_{2}\left(K_{1} E^{T}+K_{2} U^{T}\right)\right\}<0 .
$$

Note that (12) is an LMI, however, this inequality does not take into account all the information since it 
ignores matrix $C_{2}$. Thus, pre- and postmultiplying (11) by $\left(P E^{T}+V S U^{T}\right)^{-T}$ and $\left(P E^{T}+V S U^{T}\right)^{-1}$ leads to the following dual LMI:

$$
H e\left\{\left(Q E+U R V^{T}\right)^{T}\left(A+B_{2} F C_{2}\right)\right\}<0
$$

where $Q$ and $R$ satisfy (7) and (8).

Following the same lines as above, we can set: $L_{1}:=Q B_{2} F$ and $L_{2}:=R^{T} U^{T} B_{2} F$ and transform (13) to

$$
H e\left\{\left(Q E+U R V^{T}\right)^{T} A+\left(E^{T} L_{1}+V L_{2}\right) C_{2}\right\}<0
$$

However, equalities (7) and (8) are not linear. According to the idea of the cone complementary linearization method [17], (7) can be relaxed to an LMI as follows. Minimizing a linearized version of $\operatorname{trace}\left(E_{L}^{T} Q E P E_{R}\right)$ under the following LMI:

$$
\left[\begin{array}{cc}
E_{R}^{T} P E_{R} & I \\
\bullet & E_{L}^{T} Q E_{L}
\end{array}\right] \geq 0 .
$$

On the other hand, (8) can be relaxed by minimizing the following norm:

$$
\left\|S_{i+1}-S_{i}\right\|_{F}:=\operatorname{trace}\left(\left(S_{i+1}-S_{i}\right)^{T}\left(S_{i+1}-S_{i}\right)\right)
$$

and fixing $R_{i+1}=\left(U^{T} U\right)^{-1} S_{i}^{-1}\left(V^{T} V\right)^{-1}$, where the superscript $i$ means the number of iteration. This can be done by minimizing trace $(Y)$ under the following constraint:

$$
\left[\begin{array}{cc}
Y & \left(S_{i+1}-S_{i}\right)^{T} \\
\bullet & I
\end{array}\right]>0 .
$$

Based on this, an iterative algorithm is proposed hereafter to find an initial pair $\left(P_{s}, S_{s}\right)$.

Algorithm 6. We have the following steps.

Step 1. Set $i=1, P_{0}=I, S_{0}=I$, and $R_{1}=$ $\left(U^{T} U\right)^{-1} S_{0}^{-1}\left(V^{T} V\right)^{-1}$. Choose the computation parameter $\mu>0$.

Step 2. Solve the following optimization problem for $P_{i}, Q_{i}, S_{i}$ and $K_{1}, K_{2}, L_{1}, L_{2}$. Minimize $\operatorname{trace}\left(E_{L}^{T} Q_{i} E P_{i-1} E_{R}+\right.$ $\left.E_{L}^{T} Q_{i-1} E P_{i} E_{R}\right)+\mu \operatorname{trace}(Y)$ subject to the following LMI constraints:

$$
\begin{gathered}
H e\left\{A\left(P_{i} E^{T}+V S_{i} U^{T}\right)+B_{2}\left(K_{1} E^{T}+K_{2} U^{T}\right)\right\}<0 \\
H e\left\{\left(Q_{i} E+U R_{i} V^{T}\right)^{T} A+\left(E^{T} L_{1}+V L_{2}\right) C_{2}\right\}<0 \\
{\left[\begin{array}{cc}
E_{R}^{T} P_{i} E_{R} & I \\
\bullet & E_{L}^{T} Q_{i} E_{L}
\end{array}\right]>0, P_{i}>0, Q_{i}>0,} \\
{\left[\begin{array}{cc}
Y_{i} & \left(S_{i}-S_{i-1}\right)^{T} \\
\bullet & I
\end{array}\right]>0 .}
\end{gathered}
$$

Step 3. If $\operatorname{trace}\left(E_{L}^{T} Q_{i} E P_{i} E_{R}\right)-r<\varepsilon_{1}$ and trace $\left(Y_{i}\right)<$ $\varepsilon_{2}$, where $\varepsilon_{1}, \varepsilon_{2}$ some prescribed tolerances, initial variables $P_{s}$ and $S_{s}$ are found, stop.

Step 4. If the difference of two iterations is small that is $\operatorname{trace}\left(E_{L}^{T} Q_{i} E P_{i} E_{R}\right)-\operatorname{trace}\left(E_{L}^{T} Q_{i-1} E P_{i-1} E_{R}\right)<\varepsilon_{3}$ and $\operatorname{trace}\left(Y_{i}\right)-\operatorname{trace}\left(Y_{i-1}\right)<\varepsilon_{4}$ where $\varepsilon_{3}, \varepsilon_{4}$ are some prescribed tolerances, initial variables $P_{s}$ and $S_{s}$ may not be found, stop.

Step 5. Set $i=i+1$, go back to Step 2 .

Recall that the closed-loop system (10) is admissible and all the finite eigenvalues of the pair $\left(E, A+B_{2} F C_{2}\right)$ are in the strict left side of the line $\alpha / 2$ in the complex plane if and only if the following condition:

$$
H e\left\{\left(A+B_{2} F C_{2}\right)\left(P E^{T}+V S U^{T}\right)\right\}-\alpha E P E^{T}<0
$$

holds for a definite positive matrix $P \in \mathbb{R}^{n \times n}$ and a matrix $S \in \mathbb{R}^{(n-r) \times(n-r)}$. This condition will be used in Algorithm 7 to find a SOF gain ensuring admissibility of (10).

Algorithm 7. We have the following steps.

Step 1. Set $i=1, P_{1}=P_{s}$ and $S_{1}=S_{s}$, as obtained from Algorithm 6.

Step 2. Solve the following optimization problem for $F$ with given $P_{i}, S_{i}$.

Minimize $\alpha_{\mathrm{i}}$ subject to the following LMI constraints:

$$
\begin{gathered}
P_{i}>0, \\
H e\left\{\left(A+B_{2} F C_{2}\right)\left(P_{i} E^{T}+V S_{i} U^{T}\right)\right\}-\alpha_{i} E P_{i} E^{T}<0 .
\end{gathered}
$$

Step 3. If $\alpha_{i} \leq 0, F$ ensures admissibility of the closed-loop (10), stop.

Step 4. Set $i=i+1$. Solve the optimization problem (20) for $P_{i}, S_{i}$ with given $F$.

Step 5. If $\alpha_{i} \leq 0, F$ ensures admissibility of the closed-loop (10), stop.

Step 6. Solve the following optimization problem for $P_{i}, S_{i}$ with given $F$ and $\alpha_{i}$.

Minimize trace $\left(P_{i}\right)$ subject to the LMI constraints (20).

Step 7. If $\left\|P_{i}-P_{i-1}\right\| /\left\|P_{i}\right\|<\varepsilon$, a prescribed tolerance, a solution may not be found, stop, else set $i=i+1$; go back to Step 2. 
Remarks 8. (i) To shed light on the role of the computation parameter $\mu$, let us say roughly that by increasing $\mu$ we can reduce more rapidly the difference between $\left(E_{R}^{T} P E_{R}\right)^{-1}$ and $E_{L}^{T} Q E_{L}$. On the other hand, by decreasing $\mu$ we can reduce more rapidly the difference between $R$ and $\left(U^{T} U\right)^{-1} S^{-1}\left(V^{T} V\right)^{-1}$.

(ii) In Algorithm 6, the selection of parameters $\varepsilon_{1}$ and $\varepsilon_{2}$ is not crucial since the variables $P_{s}$ and $S_{s}$ are not necessarily feasible solutions of (11) but only some suitable initializing solutions. The feasible solutions will be derived by Algorithm 7.

(iii) The discussion on the iterative procedure and convergence, particularly, of Algorithm 7 follow those in [13].

(iv) As iterative LMI methods, when the proposed procedure fails to find a solution it does not mean that no solution can be found.

Example 9. Consider the SOF admissibility control problem of system (1) with $w=0$ and the following data:

$$
\begin{array}{cc}
E=\left[\begin{array}{lllll}
1 & 0 & 0 & 0 & 0 \\
0 & 1 & 0 & 0 & 0 \\
1 & 0 & 1 & 0 & 0 \\
0 & 0 & 0 & 0 & 0 \\
0 & 0 & 0 & 0 & 0
\end{array}\right], & A=\left[\begin{array}{ccccc}
1 & -3 & -3 & 1 & 6 \\
0 & -2 & 7 & 0 & 0 \\
-5 & 1 & 0 & 5 & 8 \\
0 & 0 & 6 & 0 & 0 \\
-6 & 5 & 4 & 8 & 0
\end{array}\right], \\
B_{2} & =\left[\begin{array}{cc}
1 & 2 \\
0 & 2 \\
5 & 1 \\
2 & 1 \\
1 & -3
\end{array}\right],
\end{array}
$$

The open-loop system is not admissible (unstable finite dynamics and impulsive behavior). Note that the Algorithm given in [10] could not find a solution for this system. Algorithm 6 yields initial variables $P_{s}$ and $S_{s}$ after 2 iterations. Then, an SOF gain $F$ is found as

$$
F=\left[\begin{array}{cc}
-6.7231 & 2.9436 \\
0.0571 & -0.1467
\end{array}\right]
$$

using Algorithm 7 after 3 iterations. In this case, $\alpha=$ -5.5599 and the closed-loop pair $\left(E, A+B_{2} F C_{2}\right)$ has 3 finite eigenvalues: $\{-8694.5 ;-6.2 ;-2.8\}$.
Example 10. Consider the SOF admissibility control problem of (1) with $w=0$ and the following data:

$$
\begin{array}{cc}
E=\left[\begin{array}{llllll}
1 & 0 & 0 & 0 & 0 & 0 \\
0 & 1 & 0 & 0 & 0 & 0 \\
1 & 0 & 1 & 0 & 0 & 0 \\
0 & 0 & 0 & 1 & 0 & 0 \\
0 & 0 & 0 & 0 & 0 & 0 \\
0 & 0 & 0 & 0 & 0 & 0
\end{array}\right], \quad A=\left[\begin{array}{cccccc}
1 & -3 & -3 & 2 & 1 & 6 \\
9 & -2 & 7 & 7 & 0 & 0 \\
-5 & 1 & -3 & -1 & 5 & 8 \\
3 & 0 & 6 & -1 & 9 & 0 \\
4 & -2 & 0 & -2 & -1 & 0 \\
-6 & 5 & 4 & -2 & 8 & 0
\end{array}\right], \\
B_{2}=\left[\begin{array}{ccc}
6 & 1 & 2 \\
1 & 0 & 9 \\
5 & 1 & -1 \\
9 & 1 & 2 \\
1 & -3 & 5 \\
4 & 9 & 8
\end{array}\right], C_{2}=\left[\begin{array}{cccccc}
1 & 0 & -1 & 3 & 4 \\
0 & 1 & 4 & 8 & -7 & 5
\end{array}\right] .
\end{array}
$$

The open-loop system is clearly not admissible. Algorithm 6 yields initial variables $P_{s}$ and $S_{s}$ after 2 iterations. Using Algorithm 7 after 5 iterations, an SOF gain $F$ is found as

$$
F=\left[\begin{array}{cc}
4.0683 & 0.7716 \\
2.7622 & 0.5292 \\
-7.2871 & -1.3928
\end{array}\right]
$$

In this case, $\alpha=-0.3943$ and the closed-loop pair $(E, A+$ $B_{2} F C_{2}$ ) has 4 finite eigenvalues:

$$
\{-0.7328 \pm 7.7547 i ;-1.0350 \pm 3.3930 i\} .
$$

3.2. An LMI-Based Algorithm for the Structured SOF Admissibility Control Problem. In this section, the structured SOF admissibility control problem is investigated. The structure constraint on the SOF controller is defined by

$$
F=\Lambda_{F} \otimes F, \quad \Lambda_{F} \in\{0,1\}^{m \times p},
$$

where $\Lambda_{F} \in\{0,1\}^{m \times p}$.

It is easy to see that

$$
\Omega_{\Lambda_{F}}:=\left\{F \in \mathbb{R}^{m \times p} / F=\Lambda_{F} \otimes F, \Lambda_{F} \in\{0,1\}^{m \times p}\right\}
$$

is a convex set.

The main idea underlying Algorithm 11 comes from an obvious fact: any SOF gain $F_{s}$ solving the SOF admissibility control problem may be decomposed as

$$
F_{s}=\Lambda_{F} \otimes F_{s}+\tilde{\Lambda}_{F} \otimes F_{s}=F_{1}+F_{2}
$$

where $\tilde{\Lambda}_{F}=\Gamma_{m \times p}-\Lambda_{F}$. A structured SOF (associated to the desired structure represented by $\Lambda_{F}$ ) may then be obtained when annulling $F_{2}$.

Algorithm 11. We have the following steps. 
Step 1. Set $F=F_{s}$ ( $F_{s}$ is the SOF gain obtained by Algorithm 7) and find matrices $P_{1}>0$ and $S_{1}$ such that

$$
H e\left\{\left(A+B_{2} F_{s} C_{2}\right)\left(P_{1} E^{T}+V S_{1} U^{T}\right)\right\}<0
$$

holds.

Step 2. Solve the following optimization problem for $F_{1}$ and $F_{2}$ with given $P_{i}$ and $S_{i}$.

$$
\begin{gathered}
\min _{F_{1}, F_{2}} \beta, \\
\operatorname{He}\left\{\left(A+B_{2}\left(F_{1}+F_{2}\right) C_{2}\right)\left(P_{i} E^{T}+V S_{i} U^{T}\right)\right\}<0, \\
\operatorname{trace}\left(F_{2}^{T} F_{2}\right)<\beta, \quad F_{1}=\Lambda_{F} \otimes F_{1}, \quad F_{2}=\tilde{\Lambda}_{F} \otimes F_{2} .
\end{gathered}
$$

Step 3. If $\beta<\varepsilon_{5}$, a prescribed tolerance, a feasible solution is found and ( $\left.E, A+B_{2} F_{1} C_{2}\right)$ is admissible, stop.

Step 4. Solve the following optimization problem for $P_{i}, S_{i}$ with given $F_{1}, F_{2}$ :

$$
\begin{gathered}
\min \operatorname{trace}\left(P_{i}\right), \\
P_{i}>0, \\
H e\left\{\left(A+B_{2}\left(F_{1}+F_{2}\right) C_{2}\right)\left(P_{i} E^{T}+V S_{i} U^{T}\right)\right\}<0 .
\end{gathered}
$$

Step 5. If $\left\|P_{i}-P_{i-1}\right\| /\left\|P_{i}\right\|<\varepsilon$, a prescribed tolerance, a solution may not be found, stop. Else set $i=i+1$, go back to Step 2.

Example 12. Consider the structured SOF admissibility control problem of (1) with $w=0$ and the following data:

$$
\begin{gathered}
E=\left[\begin{array}{llll}
1 & 0 & 0 & 0 \\
0 & 1 & 0 & 0 \\
0 & 0 & 0 & 0 \\
0 & 0 & 0 & 0
\end{array}\right], \quad A=\left[\begin{array}{cccc}
1 & 2 & -1 & 0 \\
-4 & 2 & 0 & 2 \\
0 & -1 & 3 & -9 \\
0 & -4 & 0 & 0
\end{array}\right], \\
B_{2}=\left[\begin{array}{ll}
2 & 1 \\
1 & 0 \\
0 & 0 \\
1 & 5
\end{array}\right], \\
C_{2}=\left[\begin{array}{cc}
1 & 0 \\
0 & -1 \\
2 & 0 \\
0 & 1
\end{array}\right], \quad \Lambda_{F}=\left[\begin{array}{ll}
1 & 0 \\
0 & 1
\end{array}\right] .
\end{gathered}
$$

Table 1 summarizes the results obtained when applying successively the proposed three algorithms. The notation $l+k$ in Table 1 means that $l$ is the iteration number of Algorithm 6 and $k$ is the iteration number of Algorithm 7.

\section{SOF $\mathrm{H}_{\infty}$ control}

The objective of the SOF $\mathrm{H}_{\infty}$ control problem is to find an SOF controller $F \in \mathbb{R}^{m \times p}$ such that the closed-loop system given by

$$
\begin{aligned}
E_{c} \dot{x}_{c} & =A_{c} x_{c}+B_{c} w \\
z & =C_{c} x_{c}
\end{aligned}
$$

with $A_{c}=A+B_{2} F C_{2}, B_{c}=B_{1}, C_{c}=C_{1}$ is admissible and satisfies the $\mathrm{H}_{\infty}$ norm constraint:

$$
\left\|C_{c}\left(s E_{c}-A_{c}\right)^{-1} B_{c}\right\|_{\infty}<\gamma \quad \text { for } \gamma>0 .
$$

According to Lemma 3, this is possible if and only if there exist a positive definite matrix $P$ and a matrix $S$ such that

$$
\left[\begin{array}{cc}
H e\left\{A_{c}\left(P E^{T}+V S U^{T}\right)\right\}+B_{c} B_{c}^{T} & \left(P E^{T}+V S U^{T}\right)^{T} C_{c}^{T} \\
\bullet & -\gamma^{2} I
\end{array}\right]<0 .
$$

As done for the SOF admissibility control problem, we propose here two iterative LMI algorithms, namely, Algorithms 13 and 14 that are similar to Algorithms 6 and 7. The main idea is to find first initial variables $P_{s}$ and $S_{s}$ and based on them derive a feasible solution $F_{s}$.

We sum up briefly the construction of these algorithms in the following.

Algorithm 13. It is constructed by using the LMI constraints:

$$
\begin{gathered}
{\left[\begin{array}{cc}
\mathcal{A}+B_{1} B_{1}^{T} & \left(P_{i} E^{T}+V S_{i} U^{T}\right)^{T} C_{1}^{T} \\
\bullet & -\gamma^{2} I
\end{array}\right]<0,} \\
{\left[\begin{array}{cc}
\mathcal{B}+C_{1}^{T} C_{1} & \left(Q_{i} E+U R_{i} V^{T}\right)^{T} B_{1} \\
\bullet & -\gamma^{2} I
\end{array}\right]<0,} \\
{\left[\begin{array}{cc}
E_{R}^{T} P_{i} E_{R} & I \\
\bullet & E_{L}^{T} Q_{i} E_{L}
\end{array}\right]>0, \quad P_{i}>0, Q_{i}>0,} \\
{\left[\begin{array}{cc}
Y_{i} & \left(S_{i}-S_{i-1}\right)^{T} \\
\bullet & I
\end{array}\right]>0,}
\end{gathered}
$$

where $\mathcal{A}=H e\left\{A\left(P_{i} E^{T}+V S_{i} U^{T}\right)+B_{2}\left(K_{1} E^{T}+K_{2} U^{T}\right)\right\}$ and $\mathscr{B}=\operatorname{He}\left\{\left(Q_{i} E+U R_{i} V^{T}\right)^{T} A+\left(E^{T} L_{1}+V L_{2}\right) C_{2}\right\}$, instead of constraints (18) in Algorithm 6.

Algorithm 14. It is constructed by using the LMI constraints:

$$
\begin{gathered}
P_{i}>0 \\
{\left[\begin{array}{cc}
\Theta & \left(P_{i} E^{T}+V S_{i} U^{T}\right)^{T} C_{1}^{T} \\
\bullet & -\gamma^{2} I
\end{array}\right]<0}
\end{gathered}
$$

with $\Theta:=H e\left\{\left(A+B_{2} F C_{2}\right)\left(P_{i} E^{T}+V S_{i} U^{T}\right)+B_{2}\left(K_{1} E^{T}+\right.\right.$ $\left.\left.K_{2} U^{T}\right)\right\}+B_{1} B_{1}^{T}-\alpha_{i} E P_{i} E^{T}$ instead of constraints (20) in Algorithm 7. 
TABLE 1: A structured SOF admissibility control problem.

\begin{tabular}{|c|c|c|c|c|}
\hline Algorithm & Iterations number & \multicolumn{3}{|c|}{ The obtained SOF } \\
\hline Algorithms 6 and 7 & $1+3$ & $F_{s}=$ & $\begin{array}{c}7.5519 \\
-1.5082\end{array}$ & $\begin{array}{c}-45.2884 \\
9.0436\end{array}$ \\
\hline Algorithm 11 & 7 & $F_{1}=$ & {$\left[\begin{array}{c}0.7509 \\
0\end{array}\right.$} & $\begin{array}{c}0 \\
-0.8472\end{array}$ \\
\hline
\end{tabular}

Example 15. Consider the SOF $\mathrm{H}_{\infty}$ control problem of (1) with $\gamma=1$ and the following data:

$$
\begin{array}{cc}
E=\left[\begin{array}{lll}
3 & 0 & 0 \\
0 & 2 & 0 \\
0 & 0 & 0
\end{array}\right], \quad A=\left[\begin{array}{ccc}
-2 & -1 & 1 \\
0 & -1 & 0 \\
1 & 1 & 0
\end{array}\right], \quad B_{1}=\left[\begin{array}{c}
0 \\
1 \\
-1
\end{array}\right], \\
C_{1}=\left[\begin{array}{c}
0 \\
0 \\
-1
\end{array}\right]^{T}, \quad B_{2}=\left[\begin{array}{c}
0 \\
1 \\
-1
\end{array}\right], \quad C_{2}=\left[\begin{array}{l}
0 \\
1 \\
1
\end{array}\right]^{T} .
\end{array}
$$

Algorithm 13 yields initial variables $P_{s}$ and $S_{s}$ after one iteration. Then, an SOF gain $F$ satisfying $\gamma=1$ is found as $F=2.4162$ using Algorithm 14 after 3 iterations. In this case, $\alpha=-0.2763$ and the closed-loop pair $\left(E, A+B_{2} F C_{2}\right)$ has two finite eigenvalues: $\{-0.2521 \pm 0.4342 i\}$.

Following the same lines as in Algorithm 11, we can propose an algorithm associated to the structured SOF $\mathrm{H}_{\infty}$ control problem. Let us denote it Algorithm 6. (For brevity reasons this algorithm is not given here, however it can be easily constructed using Algorithm 11.)

Example 16. Consider the structured SOF $\mathrm{H}_{\infty}$ control problem of (1) with $\gamma=1$ and the following data:

$$
\begin{gathered}
E=\left[\begin{array}{lll}
1 & 0 & 0 \\
0 & 1 & 0 \\
0 & 0 & 0
\end{array}\right], \quad A=\left[\begin{array}{ccc}
1 & 0 & -10 \\
0 & -8 & -1 \\
10 & 0 & 0
\end{array}\right], \\
B_{1}=\left[\begin{array}{l}
1 \\
0 \\
1
\end{array}\right], \quad C_{1}=\left[\begin{array}{lll}
0 & 1 & 1
\end{array}\right], \\
B_{2}=\left[\begin{array}{ll}
2 & 1 \\
1 & 0 \\
0 & 1
\end{array}\right], \quad C_{2}=\left[\begin{array}{ccc}
1 & 0 & 2 \\
1 & 0 & -1
\end{array}\right], \quad \Lambda_{F}=\left[\begin{array}{ll}
1 & 0 \\
0 & 1
\end{array}\right] .
\end{gathered}
$$

Table 2 summarizes the results obtained for this example.

\section{5. $\mathrm{H}_{\infty}$ Observer-Based Control for Descriptor Systems}

In this section, an alternative technique for determining observer-based $\mathrm{H}_{\infty}$ controllers for descriptor systems is discussed. Motivated by the practical appeal of such forms, many papers have dealt with this topic in the state-space case (see, e.g., [19] or [20] and references therein). The following results can be seen as an extension to the descriptor case of those derived in the state-space case.

Consider the descriptor system (1) under Assumption 5 and the observer-based feedback controller described by

$$
\begin{gathered}
E \dot{\hat{x}}=A \hat{x}+B_{2} u+L\left(y-C_{2} \hat{x}\right), \\
u=K \hat{x},
\end{gathered}
$$

The closed-loop system is given by

$$
\begin{gathered}
E_{c l} \dot{x}_{c l}=A_{c l} x_{c l}+B_{c l} w, \\
z=C_{c l} x_{c l},
\end{gathered}
$$

where

$$
\begin{aligned}
& E_{c l}=\left[\begin{array}{ll}
E & 0 \\
0 & E
\end{array}\right], \quad A_{c l}=\left[\begin{array}{cc}
A+B_{2} K & -B_{2} K \\
0 & A-L C_{2}
\end{array}\right], \\
& B_{c l}=\left[\begin{array}{l}
B_{1} \\
B_{1}
\end{array}\right],
\end{aligned}
$$

The suboptimal observer-based $\mathrm{H}_{\infty}$ control problem is to find an observer gain $L$ and a feedback gain $K$ such that the closed-loop (42) is admissible and satisfies the $\mathrm{H}_{\infty}$ norm constraint:

$$
\left\|C_{c l}\left(s E_{c l}-A_{c l}\right)^{-1} B_{c l}\right\|_{\infty}<\gamma \quad \text { for } \gamma>0
$$

Let us introduce the following augmented model:

$$
\left(\Sigma_{a}\right)\left\{\begin{array}{l}
E_{a} \dot{x}_{a}=A_{a} x_{a}+B_{a 1} w+B_{a 1} u_{a}, \\
z=C_{a 1} x_{a}, \\
y_{a}=C_{a 2} x_{a}
\end{array}\right.
$$


TABLE 2: A structured SOF $\mathrm{H}_{\infty}$ control problem $(\gamma=1)$.

\begin{tabular}{lcc}
\hline Algorithm & Iterations number & The obtained SOF \\
\hline Algorithms 13 and 14 & $1+7$ & $F=\left[\begin{array}{cc}-3.1507 & -8.2052 \\
1.2616 & 3.2928\end{array}\right]$ \\
6 & 2 & $F=\left[\begin{array}{cc}0.4677 & 0 \\
0 & 1.6957\end{array}\right]$ \\
\hline
\end{tabular}

where

$$
\begin{aligned}
& E_{a}=E_{c l}, \quad A_{a}=\left[\begin{array}{cc}
A & 0 \\
0 & A
\end{array}\right], \quad B_{a 1}=B_{c l}, \\
& B_{a 2}=\left[\begin{array}{cc}
B_{2} & 0 \\
0 & I
\end{array}\right], \quad C_{a 1}=\left[\begin{array}{ll}
C_{1} & 0
\end{array}\right], \quad C_{a 2}=\left[\begin{array}{cc}
I & -I \\
0 & C_{2}
\end{array}\right]
\end{aligned}
$$

and the structured SOF gain:

$$
F_{s s}:=\left[\begin{array}{cc}
K & 0 \\
0 & L
\end{array}\right] .
$$

The observer-based $\mathrm{H}_{\infty}$ control problem given above is equivalent to finding a structured $\mathrm{SOF} \mathrm{H}_{\infty}$ gain of the form (47) such that the closed-loop $F_{l}\left(\Sigma_{a}, F_{s s}\right)$ is admissible and satisfies the $\mathrm{H}_{\infty}$ norm constraint:

$$
\left\|F_{l}\left(\Sigma_{a}, F_{s s}\right)\right\|_{\infty}<\gamma
$$

Thus, using the proposed Algorithms 13, 14, and 6 in the previous section one can solve the aforementioned problem (i.e., $\mathrm{H}_{\infty}$ control problem for descriptor systems).

Example 17. Consider the observer-based $\mathrm{H}_{\infty}$ control problem of (1) with $\gamma=1$ for the descriptor system given in Example 15. The problem consists in finding a structured $\mathrm{H}_{\infty}$ SOF with the following fixed structure:

$$
\Lambda_{F}=\left[\begin{array}{lll|l}
1 & 1 & 1 & 0 \\
\hline 0 & 0 & 0 & 1 \\
0 & 0 & 0 & 1 \\
0 & 0 & 0 & 1
\end{array}\right],
$$

for an augmented model constructed following (45), (46), and (47). After 2 iterations of Algorithm 13, 3 iterations of Algorithm 5 and finally 9 iterations of Algorithm 6, we obtain the following admissible structured SOF gain:

$$
F_{1}=\left[\begin{array}{cccc}
5.9527 & 2.3611 & 16.7426 & 0 \\
0 & 0 & 0 & -1.2904 \\
0 & 0 & 0 & 12.0856 \\
0 & 0 & 0 & -11.9409
\end{array}\right] .
$$

\section{Conclusion}

In this note, the static output feedback synthesis problem for descriptor systems is considered. Some LMI-based algorithms to find potentially structured SOF gains ensuring admissibility and $\mathrm{H}_{\infty}$ performance of the closed-loop system are proposed. These algorithms are used in order to propose a (descriptor) observer-based $\mathrm{H}_{\infty}$ controller design method. Although iterative the proposed approaches are appealing and find suitable solutions for some benchmarking numerical examples.

\section{References}

[1] L. Dai, Singular Control Systems, Lectures Notes in Control and Information Sciences, Springer, Berlin, Germany, 1989.

[2] M. Ikeda, T. W. Lee, and E. Uezato, "A strict LMI condition for $\mathrm{H}_{2}$ control of descriptor systems," in Proceedings of the IEEE Conference on Decision and Control, vol. 1, pp. 601-604, Sydney, Australia, 2000.

[3] J. Y. Ishihara and M. H. Terra, "On the Lyapunov theorem for singular systems," IEEE Transactions on Automatic Control, vol. 47, no. 11, pp. 1926-1930, 2002.

[4] F. L. Lewis, "A survey of linear singular systems," Circuits, Systems, and Signal Processing, vol. 5, no. 1, pp. 3-36, 1986.

[5] I. Masubuchi, Y. Kamitane, A. Ohara, and N. Suda, " $\mathrm{H}_{\infty}$ control for descriptor systems: a matrix inequalities approach," Automatica, vol. 33, no. 4, pp. 669-673, 1997.

[6] E. Uezato and M. Ikeda, "Strict LMI conditions for stability, robust stabilization, and $\mathrm{H}_{\infty}$ control of descriptor systems," in Proceedings of the 38th IEEE Conference on Decision and Control (CDC '99), pp. 4092-4097, Phoenix, Ariz, USA, December 1999.

[7] S. Xu and J. Lam, Robust Control and Filtering of Singular Systems, Lecture Notes in Control and Information Sciences, Springer, Berlin, Germany, 2006.

[8] A. Trofino-Neto and V. Kucera, "Stabilization via static output feedback," IEEE Transactions on Automatic Control, vol. 38, no. 5, pp. 764-765, 1993.

[9] J.-T. Yu, "A convergent algorithm for computing stabilizing static output feedback gains," IEEE Transactions on Automatic Control, vol. 49, no. 12, pp. 2271-2275, 2004.

[10] C.-H. Kuo and C.-H. Fang, "An LMI approach to admissibilization of uncertain descriptor systems via static output feedback," in Proceedings of the American Control Conference, pp. 5104-5109, June 2003.

[11] D. L. Chu and D. W. C. Ho, "Necessary and sufficient conditions for the output feedback regularization of descriptor systems," IEEE Transactions on Automatic Control, vol. 44, no. 2, pp. 405-412, 1999.

[12] V. Lovass-Nagy, D. L. Powers, and R. J. Schilling, "On regularizing descriptor systems by output feedback," IEEE Transactions on Automatic Control, vol. 39, no. 7, pp. 15071509, 1994. 
[13] A. Fujimori, "Optimization of static output feedback using substitutive LMI formulation," IEEE Transactions on Automatic Control, vol. 49, no. 6, pp. 995-999, 2004.

[14] M. Yagoubi and P. Chevrel, "Structured $\mathrm{H}_{2}$ controller synthesis via a dilated LMI based algorithm," in Proceedings of the 44th IEEE Conference on Decision and Control, and the European Control Conference (CDC-ECC'05), pp. 5746-5751, December 2005.

[15] Y. He and Q.-G. Wang, "An improved ILMI method for static output feedback control with application to multivariable PID control," IEEE Transactions on Automatic Control, vol. 51, no. 10, pp. 1678-1683, 2006.

[16] M. Yagoubi and P. Chevrel, "An ILMI approach to structure constrained control design," in Proceedings of the European Control Conference, Porto, Portugal, 2001.

[17] L. El Ghaoui, F. Oustry, and M. Aït Rami, "A cone complementarity linearization algorithm for static output-feedback and related problems," IEEE Transactions on Automatic Control, vol. 42, no. 8, pp. 1171-1176, 1997.

[18] E. Uezato and M. Ikeda, "Strict LMI conditions for stability, robust stabilization, and $\mathrm{H}_{\infty}$ control of descriptor systems," in Proceedings of the 38th IEEE Conference on Decision and Control (CDC '99), pp. 4092-4097, Phoenix, Ariz, USA, December 1999.

[19] D. Alazard and P. Apkarian, "Exact observer-based structures for arbitrary compensators," International Journal of Robust and Nonlinear Control, vol. 9, no. 2, pp. 101-118, 1999.

[20] M. Yagoubi and P. Chevrel, "Direct versus Indirect observerbased $\mathrm{H}_{\infty}$ control," in Proceedings of the IFAC World Congress, Barcelona, Spain, 2002. 

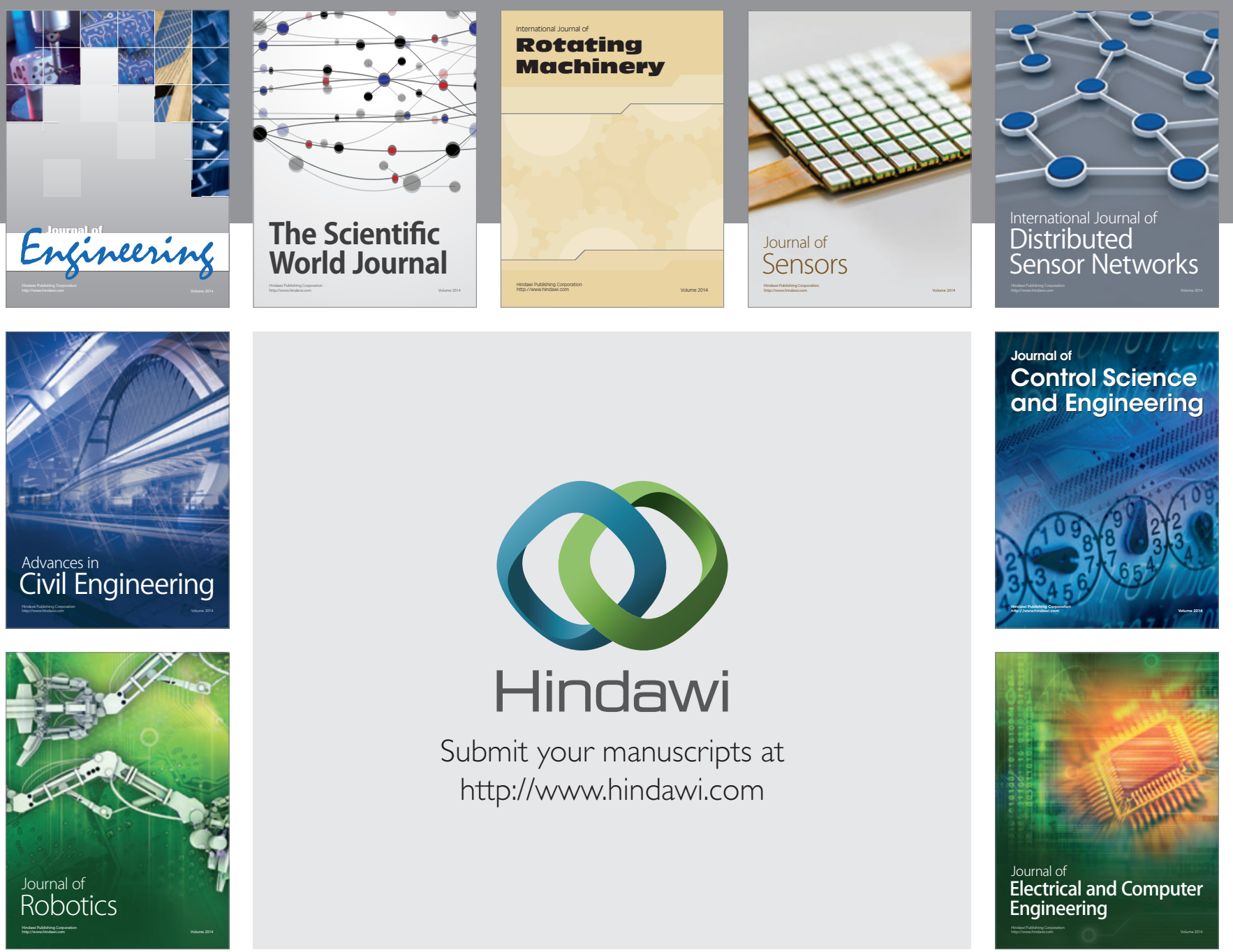

Submit your manuscripts at

http://www.hindawi.com
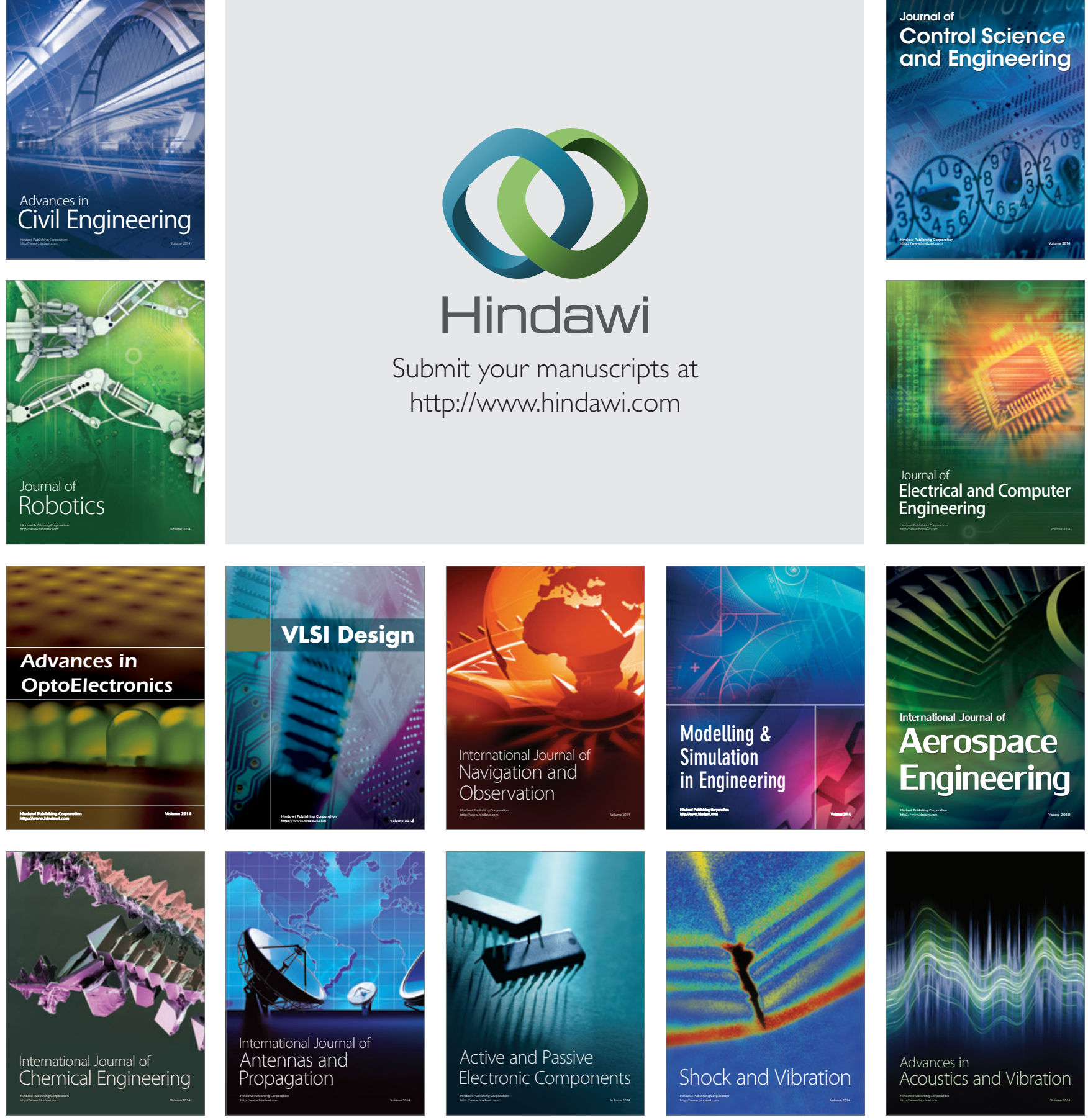